\title{
Contribution of Modeling Approaches and Virtual Populations in Transposing the Results of Clinical Trials into Real Life and in Enlightening Public Health Decisions
}

\author{
François Gueyffier ${ }^{1}$, Catherine Brun Strang ${ }^{2}$, Gilles Berdeaux ${ }^{3}$, Lionel Riou França ${ }^{4}$, Patrick Blin ${ }^{5}$, \\ Jacques Benichou ${ }^{6}$, Jacques Massol ${ }^{7}$ and participants of Round Table Nº 6 of Giens XXVII* \\ 1 Clinical Pharmacology and Therapeutic Trials, Hospices Civils de Lyon, France \& UMR5558, CNRS and Lyon 1 University, Lyon France \\ 2 Global Medical Affairs, Sanofi R\&D, Chilly-Mazarin, France \\ 3 National Conservatory of Arts and Crafts, Paris, France \\ 4 PhiSquare, Paris, France \\ 5 CIC-P 0005 Pharmacology Service, Bordeaux Segalen University, Bordeaux, France \\ 6 Biostatistical Unit, Rouen University, Rouen, France \\ 7 Phisquare, Transplant Fondation, Paris, France; University Hospital of Besançon, Saint Jacques Hospital, Besançon, France
}

Received March $20^{\text {th }}, 2012$; accepted June $4^{\text {th }}, 2012$

\section{Keywords:}

modeling; simulation; clinical trials; public decision; virtual populations

\begin{abstract}
Modeling consists in aggregating separate pieces of knowledge, according to a given structure and rules. It allows studying the behavior of more or less complex systems by simulation techniques. Modeling is used in different state-of-theart technological domains (meteorology, aeronautics). Its use has grown for the evaluation of medicines and medical devices, from conception to prescription (marketing authorization, reimbursement, price setting and re-registrations). It follows a scientific approach and is the object of good practice recommendations. Coupling models to virtual populations allows obtaining realistic results at the population level, testing diagnostic or therapeutic strategies, as well as estimating the consequences of transposing the results of clinical trials to the population. Through examples, the participants of the Round Table analyzed the contributions of the coupling of models and realistic virtual populations, and proposed guidelines for their judicious and systematic use.
\end{abstract}

Abbreviations: see end of article.

\section{Introduction}

We all model and have done so for a long time. Upon painting a pipe and provoking the spectator of his picture by specifying: "This is not a pipe", Magritte invites us to keep in mind the permanent gap between reality and how we perceive it. Our perceptions are organized in models, more or less fragmented, limited or complex, offering us tools to support exchanges (e.g., language, spoken or written, is a model, or a set of models), allowing us to understand each other, and especially to foresee the results of our actions. Modeling through mathematical language presents remarkable advantages of power and efficiency, made accessible through the developments of computing technology. Mathematical models can achieve high levels of complexity, far away exceeding the capacity of abstraction and prediction of the human brain, prolonging its dreams of development. One of the first modeling approaches in health was used in 1760 by Bernoulli in a report to the Academy of Science in Paris, ${ }^{[1]}$ in which he proposed a model of an epidemic of smallpox, to predict the benefit and the risks of the inoculation of the disease in a population exposed to this epidemic. Formal modeling is widely used in other domains than health, such as environment with meteorology forecasts, or aeronautics to build prototypes.

\footnotetext{
* For the list of participants, see end of article.
} 
The question of transposing the results of clinical trials arises at all stages of the administrative life of drugs: in estimating the benefit to risk ratio for the market authorization, in quantifying its therapeutic interest for the access to reimbursement decision, when negotiating prices and volumes for the contract with the Comite Économique des produits de santé (Economic Committee of the Health Products, a national regulatory body in France), and at the re-registration stage. Addressing these various questions can rest on expert opinion, on a simple rule of proportionality based on simplistic hypotheses, or on models based on more complex hypotheses.

The administrative decisions regarding drugs (marketing authorization or access to reimbursement) are essentially based on data from randomized controlled trials. But it is well-known that clinical trials do not follow real life conditions. The numerous uncertainties surrounding public health decisions include not only the variability of biological phenomena, but also the transposition of trial results to real life. Helping decision-makers in predicting or quantifying the benefit expected from drugs requires modeling approaches, in order to achieve enlightened public decisions. With this in mind, the participants of the Round Table $\mathrm{N}^{\circ} 6$ settled as their objective to assess, on the basis of concrete examples, the role of modeling approaches in health, and define its conditions of acceptability to transpose the results of clinical trials and enlighten the public decision.

\section{Some key definitions}

- Transposition: application of data (e.g. results from randomized controlled trials and meta-analyses) to populations and conditions different from those under which they were obtained. ${ }^{[2]}$ N.B.: medical decisions always involve transposition and extrapolation, from what has been observed elsewhere, before, or on other individual(s) with different characteristics, in order to betters what we predict from the patients' evolution.

- Realistic virtual population: a population simulated from available knowledge (epidemiological and environmental data), to generate in silico a picture of the real population and its evolution with time. Virtual populations offer important advantages when the overall benefit to risk ratio at the population level, obtained from aggregating individual benefit to risk ratios, is not directly computable from a representative average profile, because of the complexity of the association rules and the multiplicity of the dimensions of the functions at stake. The virtual population can naturally become the support of explorations of different scenarios to use a drug or a therapeutic strategy, whether simple or complex. Depending on external constraints, such analyses of various strategies can lead to identify benefit thresholds, hence providing a reference for rational therapeutic decisions. The generation of a virtual population must aim at obtaining an in silico population as realistic as possible, and capturing the most relevant features of the real population: this defines the concept of realistic virtual population (RVP).

- Model: simplified representation of a complex real process. For example, statistical models provide a simplified description of the process of experimental results occurrence, allowing estimation and testing of parameters. For instance, they allow estimating the probability of observing these results under the null hypothesis, e.g., the hypothesis that two compared outcome distributions have the same average value. Computing the sample size of a randomized controlled trial relies on a model considering two hypotheses, the null and the alternative hypothesis, an a priori estimate of the size of the expected difference between relevant outcome parameters, the variability of measurements, and the probabilities of false positive (Type I) $\alpha$ or false negative (Type II) $\beta$ errors.

Building mathematical models is a multidisciplinary activity, one important quality criterion being reproducibility, resting on the transparency of assumptions at the various stages of model building. The resulting models are updatable, and can be used in their entirety or partially in other projects aiming at other objectives. Applied to therapeutics, this activity allows quantifying the expected effects, benefit or harm, but also the overall uncertainty surrounding the results through the estimation of the components of uncertainty at the different stages of the process. This is of high interest when dealing with complex situations. Modeling is a classical tool to treat data in the domains of pharmacokinetics and pharmacodynamics.

- Population approach: using population approaches in pharmacological modeling allows taking into account the different parts, explained and unexplained, of the overall variability when building a model. Practically speaking, building models, parameterizing them on more or less representative samples, then coupling them to more or less realistic virtual populations, can help in all stages of the development and the administrative life of drugs, before and after the approval and access to reimbursement. In particular, modeling is the only way to adequately address the needs to synthesize all the available information at a given time, whether phase I, II, III or post marketing data are concerned.

\section{Building on experience}

Numerous modeling approaches are being carried out in health, in various contexts and using various methods. Through example and situations briefly exposed below, the participants of Round Table $\mathrm{N}^{\circ} 6$ exchanged experiences and thoughts on mathematical modeling, which led them to offer proposals and suggest guidelines regarding the use of these modeling approaches in the drug approval 
process, with a special focus on issues of transposing evidence from one context to another.

\subsection{A modeling approach already well acknowledged: the example of vaccines}

Within the framework of the annual hearing of pharmaceutical firms by the Comité technique des vaccinations ${ }^{[3]}$ (Technical Committee on Vaccines, a national public health body in France), additional data with regard to the marketing authorization (MA) can be brought, including results from modeling the impact of vaccines in France. If the case is complex, a partnership with Institut de veille sanitaire (Institute for Disease Watch, a national agency in France, with missions somewhat similar to those of the Centers for disease control in the United Sates of America) is systematically recommended, with the specific aim of building a model of the impact of the vaccine in terms of morbidity and mortality or in medico-economic terms.

\subsection{Using virtual populations to measure the impact of a new treatment of septic shock}

Following the publication of results from the PROWESS trial, ${ }^{[4]}$ which showed a benefit on mortality with drotrecogin alpha in patients with septic shock, the impact of using this drug as a treatment of septic shock has been reassessed. In order to build a predictive model of the impact of this drug, the researchers combined data from PROWESS with data from a network of French intensive care units called CUB-Réa ${ }^{[5]}$ that provided information on the distributions of patient characteristics in these units (type and length of hospital stay, type of organ failures, etc.) and allowed to define sub-groups of patients. Yet other data from the medical literature allowed to more precisely estimate these distributions, e.g. when some patient strata were not enough represented in the CUB-Réa population. As a result, the virtual population issued from the combination of CUB-Réa and these additional epidemiological data became more realistic, in that it better represented the real population hospitalized in French intensive care units. This allowed estimating a realistic average effect expected from the use of the drug ${ }^{[6]}$ at the French population level.

\subsection{Virtual population and modeling to simulate long-term impact: an example in glaucoma}

Glaucoma remains a major cause of blindness in high-income countries. High intraocular pressure greatly increases the risk of glaucoma occurrence and progression. It has been shown that controlling the intraocular pressure reduces the incidence of glaucoma and slows down its progression. The control of intraocular pressure can be obtained through the use of drugs (in the form of eyes drops) or physical means (surgery, laser). The evidence leading to drug approval has relied mainly on the control of intraocular pressure. In contrast to using this intermediate outcome, using clinical outcomes such as eventual blindness would require studies with very long follow-up, which would not be compatible economically with the limited duration of patent protection. Modeling became justifiable to overcome the limitations of the regulatory process.

A first survey was set up on a randomized sample of ophthalmologist practices in France in order to collect the data necessary to parameterize the model. The risk factors of glaucoma progression were identified and quantified, as well those associated with high costs, in real situations of prescription. Treatment side effects and treatment switches were associated with a higher probability of glaucoma progression. ${ }^{[7]}$ Treatment switches and disease progression contributed to higher costs. ${ }^{[8]}$ A Markov chain stochastic model was built to aggregate the risk functions of outcome and progression. ${ }^{[9,10]}$ The temporal horizon of the model was a subject's entire life. The probability of death was modeled from the French tables of mortality. The patients could benefit from 4 lines of treatment in the form of eye drops, and could have access to surgery or laser treatment if needed; the non-responders used again the lines of eye drops, this until death. The probability of treatment failure was estimated from prescription databases such as the UK General practise research database in the United Kingdom or from clinical trial data. The results from this model were validated by contrasting them to real data and it appeared that the model was able to reproduce the results of clinical trials on long-term morbidity (e.g., the Ocular hypertension treatment study).

This approach, rather usual in health economics, allows to aggregate data from various sources, i.e., from clinical trials and from real life, allowing numerous useful extrapolations during initial drug registration, with the possibility to assess model prediction during subsequent re-registration and update the model if needed. The large number of rules of knowledge, the complexity of the rules of association and the numerous sources of uncertainties required using a stochastic approach such as Markov chain modeling and generating a virtual population.

\subsection{Virtual population to simulate cardiovascular risk prevention strategies : the example of hypertension}

Marchant et al. generated a realistic virtual French population between 35 and 64 years of age, reproducing the distribution of the relevant cardiovascular risk factors, thus allowing computing the cardiovascular risk of every individual in the generated population. The primary data source was the survey MONICA FRANCE. ${ }^{[11]}$ The age- and sex-distribution was obtained from the national statistics provided by Institut national de statistiques et études économiques 
or INSEE ${ }^{[12]}$ (National institute for statistics and economic studies, a national agency in France). The external validation included confronting the average risk scores predicted by age and sex groups to the national mortality statistics. Two cardiovascular risk scores were selected by an ad hoc working group, i.e., SCORE and Framingham. This validation step allowed illustrating the lack of internal coherence of the Framingham risk equations, the predicted coronary mortality being higher than the cardiovascular mortality in middle-aged men. The availability of information regarding every individual from the virtual population allowed analyzing this problem in detail, bringing to light too high coefficients for smoking status and the ratio between total cholesterol and high density lipoprotein cholesterol $^{[13]}$ in the coronary death score. This virtual population was an appropriate support to compare the theoretical application of the recommendations regarding antihypertensive drug treatments, ${ }^{[14]}$ and the results of a representative epidemiological survey. ${ }^{[15]}$ This comparison showed that, if the recommendations do not seem to be strictly applied regarding high blood pressure screening, they are not followed either regarding the risk level: hypertensive young women were treated far beyond what their low level of risk indicated. It was also shown that the change of threshold for defining hypertension, from 160/95 to 140/ $90 \mathrm{mmHg}$, had doubled the prevalence of hypertension, which had never been showed before; nota bene: the level of evidence regarding the benefit to risk ratio in this half of the hypertensive population is incredibly low. This illustrates the direct capacity of realistic virtual populations to estimate the size of the target populations of a drug.

The selection of the individuals to treat, according to their level of predicted risk, regularly but unsuccessfully promoted for more than 30 years through guidelines, is mathematically the most effective ${ }^{[16]}$ in terms of the number of prevented cardiovascular events. However, this strategy gives the same weight to the events occurring at 30 or 80 years. The virtual population described above allowed simulating what should be the predicted risk thresholds for prescribing antihypertensive drugs if the goal was to reduce the disparities between sex and between generations. These disparities are induced by the selection of individuals merely on their risk level, due to the heavy weight of sex and most of all age in the predicted risk. The innovative objective of this new strategy was defined as the prevention of the same proportion of events for all the age strata, and for men and women. This new strategy appeared more expensive for the same number of prevented event, but events were more often prevented in younger people: the new strategy thus prevented more years of healthy life. This example illustrates the usefulness of using realistic virtual populations as a tool to work out strategies directed towards medico-economic objectives. These strategies are complex because of their multidimensional nature: in this example, they required jointly considering the distribution of blood pressure level, the predicted risk equations, the predicted risk distribution by age and sex, and the life expectancy of the individuals. ${ }^{[17]}$

\subsection{Modeling the public health impact of a drug on multiple outcomes}

Discrete event simulation (DES) is frequently used in engineering to reproduce the behavior of complex systems. It has begun to know some success in the medical domain (346 publications in Pubmed accessed on March $17^{\text {th }}, 2012$ ). DES is a technique of simulation which consists in considering sequentially at every time $t$, over a defined time span, the future of every individual of a realistic virtual population in terms of events (e.g., myocardial infarction, second occurrence of micro-vascular infarct, complication, death, etc.) as a function of their initial characteristics such as risk factors, changes of these risk factors with time, but also therapeutic interventions (e.g., treatment exposures, side-effects of treatment, treatment switches, interruptions of treatment, hospitalizations, etc.) and previous events. ${ }^{[18,19]}$

One of the main advantages of DES is the simultaneous use of several functions or specific mathematical models for every studied event, taking into account complex and competitive situations. ${ }^{[20,21]}$ JJ Caro used DES to estimate the public health interest of rimonabant in a French obese or overweighed diabetic population. ${ }^{[18]}$ In this example, the characteristics of the virtual population were derived from French demographic data and from epidemiological studies monitoring of trends and determinants of cardiovascular diseases (MONICA), échantillon national témoin representatif des personnes diabétiques (representative national sample of people with diabetes in France or ENTRED) and étude de prise en charge du diabète de type 2 en France (study of management of type 2 diabetes in France or ECODIA). Changes in the risk factors and use of treatment over time were estimated from predictive equations according to patient characteristics and the effect of treatment was estimated from that observed in the rimonabant in obesity and related disorders (RIO Diabetes) study. ${ }^{[22]}$ The authors used Framingham equations, corrected for France as risk functions for the first cardiovascular event, the Saskatchewan equations for predicting recurrences, United Kingdom prospective diabetes study (UKPDS) scores for microvascular events, and French mortality data for death.

\section{Discussion}

A large number of questions in public health can only be answered from the use of mathematical models, validated where possible with series collected over long time periods and large geographical areas. In the context of prevention (e.g., of mortality by lipid lowering drugs, blindness by anti-glaucoma drugs, etc.), randomized clinical trials give an appropriate estimate of the average relative benefit, directly on the clinical outcome of interest, or on an intermediate outcome such as cholesterol level or intraocular pressure. If the demonstration of the benefit is made only on the 
intermediate outcome, marketing authorization can be granted assuming that the intermediate outcome gathers the properties of a good surrogate criterion. ${ }^{[23]}$ It was not in the objective of the Round Table to discuss this problem, but it has been increasingly recognized that no good surrogate criteria do exist. ${ }^{[24]}$ Two limitations to the model of surrogacy can easily be thought of: 1) mechanisms at play are often multiple and the clinical effects cannot be entirely explained by the action on the intermediate outcome; the model of prediction should thus take into account several partial intermediate outcomes to be more effective; 2) the unwanted effects obey mostly to other mechanisms that the mechanism implying the intermediate outcome. Modeling public health impact requires that the relationship between the intermediate and clinical outcomes be unambiguous, which is generally not provable with a high level of confidence. This relationship can be estimated for example through meta-regression. In what follows, we consider the more satisfactory case where the demonstration of the effect is made directly on the clinical outcome. The population studied in randomized clinical trials is well defined thanks to the eligibility criteria. When no modifier of the relative benefit of treatment can be identified, this benefit estimate can be extrapolated to any treated patient: the level of benefit is calculable for all the individuals of the treated population. In these conditions, modeling is simple, provided that i) a risk score is available; and ii) the distribution of the risk factors in the population allows defining the structure and the size of the target population. The application of the risk score and the relative benefit allow direct calculation of the number of prevented events. The same procedure applies to side-effects.

Modeling follows the complete set of specifications of any scientific research program: appropriately defining the objectives of the work, exploring the internal validity of the model, defining precisely the underlying hypotheses, exploring their level of reliability (e.g., through the analysis of the level of evidence of the trials taken into account), defining precisely and completely the methods that were used. The parameters of the equations of the model can come from the literature, or be identified by optimization processes, which are all based on the same principle: reducing the distance between the predictions of the model and the external control real data. The final validation of the model requires confronting the predictions from the model to observations that are independent from those which were used to estimate the model parameters. The processes for building medico-economic models have been formally defined through recommendations by the International Society for pharmaco-economics and outcomes research (ISPOR), ${ }^{[25]}$ and by the College of the french health economists in France.

The limitations of modeling have to be well understood and analyzed, so that the approach can take the place it deserves. Technical limitations concern in particular the availability of source data and their reliability; this issue is tightly linked to that of the level of evidence of the assumptions upon which the model is built. These limitations can be reduced if pathophysiological, epidemiological studies and clinical trials are conducted following logics enabling the

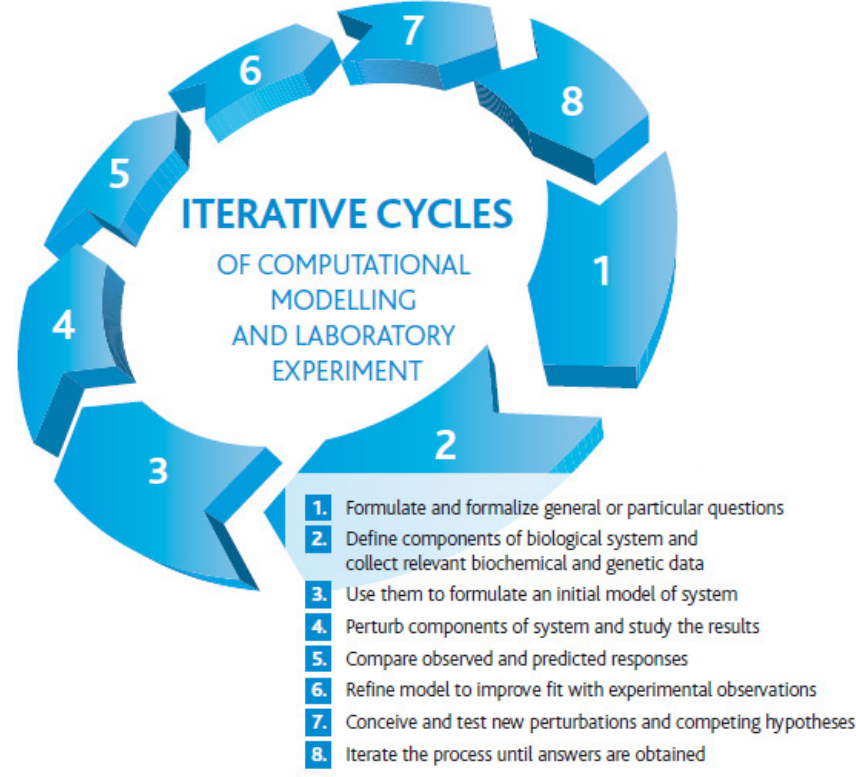

Fig. 1. The cycle of model building in interaction with external experimental or observational data. ${ }^{[26]}$ With kindly authorization of Charles Auffray (personnal communication). Available on http://www.eisbm.org/include/ pdf.eisbm_corpratebrochure.pdf.

integration of their results within pre-existing models, taking into account at their inception the current status of model developments and of remaining relevant biological questions. The development of interactions between model building and the conduct of biological and clinical experiments is essential, leading to the virtuous cycle of knowledge integration (figure 1). ${ }^{[26]}$

Of note is the generally phenomenological nature of the current model versions, which describe through mathematical functions the surface of observed phenomena, not their intimate mechanisms. Taking into account deeper levels of complexity, making the model closer to the mechanisms at play, either for the disease of for its treatment, requires the coupling of classical modeling approaches in pharmacology (pharmacokinetics, PK and pharmacodynamics, PD) with those of systems biology and physiopathology (physiologically based-, PBPK and PBPD).

Beyond its purely technical limitations, the modeling approach faces psychological obstacles: the cultural deficiency of the various actors in the development and the regulation of the drugs explains the impression of lack of transparency which they may feel when faced with results of modeling, as if they were confronted with a black box. This makes difficult any real sharing of the benefits from the approach. Furthermore, the predictive models have to be challenged in a continual confrontation to real life data: one of the best manners to make models' results acceptable is to demonstrate in real life studies that what is observed corresponds, with a reduced margin of error, to what was predicted by the model.

In addition to these cultural/psychological obstacles, the issues of conflicts of interests, although not specific to modeling 
approaches, may play a role. Such potential conflicts include the competition between model builders and experts - Key opinion leaders - who do not know or understand this approach, or the nonscientific orientation of the work, driven by financial of marketing promotion interests. So, in this domain also, it is important that the actors remain truly independent from the various interests at play, keeping as their unique final objective the public health interest. This highlights the importance of conducting modeling approaches with maximum transparency to ensure their reproducibility, with a qualified and independent scientific committee, as for studies requested by health and regulatory authorities as a whole.

Authorities in charge of reimbursement decisions can request that modeling approach be used for synthesizing available clinical trial data and allow estimating the clinical efficacy and costs-to-benefit ratio ${ }^{[27]}$ when: 1) all available data do not come from the same trial; 2) the trial participants have not the same characteristics than the patients supposed to benefit from treatment; 3) the criteria for expressing results are neither Health related quality of life nor survival; 4) no comparators were used; 5) sub-groups were not defined; 6) the long-term benefits were not observed within the follow-up period. Guidelines from the United Kingdom National Institute for health and clinical excellence (NICE) influence the decisions taken in other European countries, in the United States or even low-income countries. The lack of favorable opinion from NICE for a drug is perceived as an important barrier for succeeding in other markets. Reinforcing the modeling skills in France, as well as epidemiological and clinical research platforms with a national and international audience is a considerable stake: it could considerably strengthen the ability of French authorities to answer relevant public health questions that only mathematical models could address.

\section{Prospective considerations}

\section{1. For the actors of drug development}

The success of transposition by modeling on virtual populations requires an effective interfacing of all the key actors, who must be associated in particular in the supervision of the initiative through well formalized interactions. The various concerned entities must be represented in this supervision: marketing authorization committee, transparency commission, french High health authority (HAS), and Economic committee for health products (french Comité économique des produits de santé or CEPS), National institute of health survey (INVS), pharmaceutical industry, academic, etc. This supervision effort must be conducted in relationship with already existing working groups, national and international as well: experts from ISPOR, the harmonization working group of the estimation methods for benefit to risk ratios in files submitted to the European medical agency, the Eunet Health Technology Assessment group which are in charge of harmonizing assessment methods for reimbursement, etc.
The deliverables of such supervision are to give precise advice to drug makers when they solicit the Transparency commission, High health authority, and Economic committee for health products about the use of models in their files.

The different actors, from the pharmaceutical industry or academic world, users or deciders, must be made sensitive and trained in the underlying concepts of the modeling approach as well as its limitations, but also to its necessary use within the framework of enlightened decision-making. The expected wide-ranging use of the approach and of its concepts makes necessary the training of the physicians who are end users, whether in initial or continuous medical education. In the same spirit, the general public must be made somewhat familiar with these tools and what they can provide.

\subsection{For an academic research policy}

In view of the importance of the modeling and the necessary development of the specific tools, a significant part of epidemiological research should be devoted to the collection of information necessary to the construction of virtual populations, local or region-specific, and favor the pooling of the essential information within databases - repositories of clinical trials and of epidemiological studies, at the individual patient/participant level. The scale of the necessary developments of modeling in medicine and the construction of virtual populations justify a specific orientation of grants, e.g., writing a specific modeling line in the Programme hospitalier de recherche clinique or $\mathrm{PHRC}$ (Clinical research hospital program, a major program from the french Department of health to which physicians can apply for obtaining funding in clinical research), in the programs of the french National agency for research (Agence nationale de la recherche or ANR), or within the framework of the $\mathrm{R} \& \mathrm{D}$ framework program of the european Union. It seems necessary to encourage setting up large modeling bodies, such as the European Institute for Systems Biology and Medicine. ${ }^{[28]}$

\section{Conclusion and recommendations from the members of the Round Table}

The participants of the sixth Round Table of Giens 2011 recommend that: (i) the use of modeling be systematically considered and, if decided upon, that modeling be conducted, interpreted, validated and applied in an appropriate fashion; (ii) all the required professional skills be implemented, at all stages in view of the multidisciplinary nature of modeling and simulations; (iii) all data required for optimal modeling be made accessible through an appropriate partnership between drug makers, academics, regulators and health authorities; (iv) clinicians be made conscious of its potential contributions through acquiring experience and minimal skills at least in the interpretation of modeling outcomes; (v) experts in modeling develop algorithms or decision trees to help non-experts in 
deciding when it is useful to integrate modeling approaches in drug development; (vi) universities and high-learning institutions propose appropriate training in modeling and simulation. ${ }^{[29]}$

Several priorities could be defined, to guide research policies:

1. Methodological developments: the description of the contextual variability, in itself, and the analysis of its determinants, require important methodological developments.

2. Multidisciplinary work: the support for setting-up networks of teams combining different disciplines such as sociology, epidemiology, public health, statistics, mathematics, computer engineering also constitutes a priority. Such networks should be not only supported by specific interventions, but also particularly valued in the various research institutions themselves, e.g., by taking into account the often different scientific modalities of evaluation of these disciplines: developing interactions between these disciplines should be seen as a worthwhile accomplishment and highly encouraged, rather than perceived as a succession of obstacles without corresponding rewarding.

3. Constitution of databases: one of the major obstacles to the development of modeling in a specific geographical context lies in the paucity of individual (i.e., not aggregated) health-related data with geo-referencing at the finest possible level). If it is not per se a research work, the constitution of such databases by merging data stemming from various existing information systems (census data, disease registries, health insurance system databases, national mortality data, data on diseases with compulsory declaration, research cohorts may be regarded as some equivalent of "big equipment" programs for basic research. It is a necessary condition to develop quality research. It requires specific know-how and thus specific financing. It must be able to benefit the largest possible number of researchers. Finally, it must be transparent and accessible for the different institutional owners, and respect confidentiality constraints.

\section{Working group for the follow-up of Round Table $\mathrm{N}^{\circ} 6$ recommendations}

At last, the participants of the Round Table suggested setting up a working group who would put these recommendations into action. The timetable of this working group include i) creating a lexicon or glossary, allowing the different actors coming from different disciplines to agree upon language and concepts with a common content; ii) elaborating technical recommendations for the conduct of modeling approaches, the assessment of their quality \& validity, their integration within the process of drug development and market access; iii) the follow-up of the guidelines of Giens XXVII $6^{\text {th }}$ Round Table.

\section{Participants.}

Béatrice Augendre Ferrante (Laboratoire Lilly France), Jacques Benichou (CHU, Rouen), Gilles Berdeaux (Alcon Labs), Patrick Blin (Université de Bordeaux); Thomas Borel (Sanofi-France),
Cecile Rey-Coquais (Laboratoire Pfizer France), Jean-Michel Joubert (UCB Pharma S.A), François Meyer (Haute autorité de santé), Sophie Muller (Labaratoire GlaxoSmithKline France), Leslie Pibouleau (Haute autorité de santé), Marion Pinet (Haute autorité de santé), Christel Vidal (CHU, Besançon).

\section{Conflicts of interest. None.}

Abbreviations. CEPS: Economic committee of the health products in France (Comité économique des produits de santé); CTV: Technical committe of vaccinations in France (Comité technique des vaccinations); DES: discrete event simulation; EMA: European medicines agency; ENTRED: representative national sample of people with diabetes in France (échantillon national témoin représentatif des personnes diabétiques); ECODIA: study of management of type 2 diabetes in France (étude prise en charge du diabète de type 2); HAS: High health authority in France (Haute autorité de santé); INSEE: National institute for statistics and economic studies in France (Institut national de la statistique et des études économiques); INVS: Institute for disease watch in France (Institut de veille sanitaire); ISPOR: International Society for pharmacoeconomics and outcomes research; MA: marketing authorization; MONICA: monitoring of trends and determinants of cardiovascular diseases; PBPD: physiologically based pharmacodynamic; NICE: National Institute for Health and Clinical Excellence; PBPK: physiologically based pharmacokinetic; PHRC: clinical research hospital program in France (programme hospitalier de recherche clinique); PK/PD: pharmacokinetics/pharmacodynamics; RIO Diabetes: rimonabant in obesity and related disorders; RVP: realistic virtual population; UK GPRD : United Kingdom general practice research database; UKPDS: United Kingdom prospective diabetes study.

\section{References}

1. http://www.fyma.ucl.ac.be/files/Euler-Bernoulli.pdf

2. Massol J, Zylberman M, Goehrs JM, et al. Use of the foreign studies: transposition of the results, prediction of the therapeutic effects in the french population, modelling of the public health interest. Therapie 2006; 61: 4819, 491-9

3. Launay O, Hoch D, Saint-Sardos C, et al. Vaccines: Specific features, simulation of impact and medico-economic modelling. Therapie 2010; 65(4): 357-65

4. Bernard GR, Vincent JL, Laterre PF, et al. The Recombinant Human Activated Protein C Worldwide Evaluation in Severe Sepsis (PROWESS) Study Group, Efficacy and safety of recombinant human activated protein $\mathrm{C}$ for severe sepsis. N Engl J Med 2001, 344: 699-709

5. Aegerter P, Auvert B, Buonamico G, et al. Organization and quality control of a clinical database on intensive care medicine in central and suburban Paris. Rev Epidemiol Sante Publique 1998; 46(3): 226-37

6. Riou França L, Launois R, Le Lay K, et al. Cost-effectiveness of drotrecogin alfa (activated) in the treatment of severe sepsis with multiple organ failure. Int J Technol Assess Health Care 2006; 22(1): 101-8 
7. Denis P, Lafuma A, Berdeaux G. Medical outcomes of glaucoma therapy from a nation wide representative survey. Clin Drug Investig 2004; 24(6): 343-52

8. Denis P, Lafuma A, Berdeaux G. Medical predictive factors of glaucoma treatment costs. J Glaucoma 2004; 13(4): 283-90

9. Nordmann JP, Lafuma A, Deschaseaux C, et al. Clinical outcomes of glaucoma treatments over a patient lifetime: a Markov model. J Glaucoma 2005; 14(6): 463-9

10. Nordmann JP, Lafuma A, Berdeaux G. Modelling the lifetime economic consequences of glaucoma in France. J Med Econ 2009; 12(1): 9-16

11. Richard JL. The MONICA project. A WHO research project in cardiovascular diseases. Rev Epidemiol Sante Publique 1988; 36(4-5): 325-34

12. Institut National des Statistiques et d'Etudes Economiques. Structure par âge des populations féminine et masculine. http://www.insee.fr/

13. Marchant I, Boissel JP, Kassai B, et al. SCORE should be preferred to Framingham to predict cardiovascular death in French population. Eur J Cardiovasc Prev Rehabil 2009; 16(5): 609-15

14. Marchant I, Nony P, Cucherat M, et al. The global risk approach should be better applied in french hypertensive patients: a comparison between simulation and observation studies. PLOS One 2011; 6: e17508. doi: 10.1371/ journal.pone. 0017508

15. Godet-Thobie H, Vernay M, Noukpoape A, et al. Mean blood pressure level and prevalence of hypertension in 18 to 74 year-old adults, ENNS Survey 2006-2007: InVS; 2008. http://www.invs.sante.fr/beh/2008/49_50/ index.htm

16. Boissel JP, Kahoul R, Amsallem E, et al. Towards personalized medicine: exploring the consequences of the effect model-based approach. Personalized Medicine 2011; 8: 581-6. http://www.futuremedicine.com/doi/abs/ 10.2217/pme.11.54

17. Marchant I, Gueyffier F. Towards equitable strategies for hypertension management in cardiovascular prevention. Oral communication, EACPT Budapest 2011

18. Caro JJ. Estimation de l'intérêt de santé publique de rimonabant $20 \mathrm{mg}$ dans une population française de patients en surpoids ou obèse et diabétiques de type 2 non contrôlés: L'apport d'une modélisation. ALFEDIAM 2007, Marseille, 23 mars 2007

19. Caro JJ. Pharmacoeconomic analyses using discrete event simulation. Pharmacoeconomics 2005; 23(4): 323-32

20. Cooper K, Brailsford SC, Davies R, et al. A review of health care models for coronary heart disease interventions. Health Care Manag Sci 2006; 9(4): 311-24

21. Caro JJ, Möller J, Getsios D. Discrete event simulation: the preferred technique for health economic evaluations? Value in Health 2010; 13(8): 105660

22. Scheen AJ, Finer N, Hollander P, et al. Efficacy and tolerability of rimonabant in overweight or obese patients with type 2 diabetes: a randomised controlled study. Lancet 2006; 368: 1660-72

23. Boissel JP, Collet JP, Moleur P, et al. Surrogate endpoints, a basis for a rational approach. Eur J Clin Pharmacol 1992; 43: 235-44

24. Gueyffier F, Dib M, Boissel JP. Marqueurs biologiques : biomarqueurs - utilisation au cours du développement et pour l'enregistrement des médicaments. Therapie 2001; 56: 355-61

25. http://www.ispor.org/workpaper/research_practices/PrinciplesofGoodPracticeforDecisionAnalyticModeling-ModelingStudies.pdf

26. http://www.eisbm.org/include/pdf/eisbm_corporatebrochure.pdf

27. Guide to the Methods of NICE Technolgy Appraisals, NICE, 2008. http:// www.nice.org.uk

28. http://www.eisbm.org

29. http://mastersantepublique.univ-lyon1.fr/, spécialité PHAME (PHArmacologie, Modélisation et Essais Cliniques)

Correspondence and offprints: François Gueyffier, Clinical Pharmacology and Clinical Trials Service, Louis Pradel Hospital, BP Lyon Monchat, 69394 Lyon Cedex 03, France.

Email: francois.gueyffier@univ-lyon1.fr 